\title{
RECURRENCES AND DETERMINANTAL EXPRESSIONS FOR VALUES OF THE DIRICHLET $L$-FUNCTION
}

\author{
TAKASHI AGOH
}

\begin{abstract}
In this paper, by applying a certain Saalschütz-Gelfand type formula for generalized Bernoulli numbers, we first study shortened recurrence relations for the values of the Dirichlet $L$-function attached to a primitive character at nonpositive integers and then derive some detreminantal expressions for these values. Further, we discuss several kinds of infinite sums involving the Riemann zeta values at negative odd integers and establish some closed formulas for them by making use of special identities related to the reciprocals of central binomial numbers.
\end{abstract}

\section{INTRODUCTION AND PRELIMINARY}

The Dirichlet $L$-function and its allied zeta functions play an important role mainly in analytic number theory and they have various applications in many branches of theoretical physics, probability theory, theory of automorphic forms, applied statistics, and other areas.

Let $\chi$ be a primitive Dirichlet character with conductor $f=f_{\chi}$. The Dirichlet $L$-function $L(s, \chi)$ attached to $\chi$ is defined by the series

$$
L(s, \chi):=\sum_{n=1}^{\infty} \frac{\chi(n)}{n^{s}}, \quad s \in \mathbb{C} .
$$

This series converges absolutely and uniformly in $\operatorname{Re}(s) \geq 1+\varepsilon$ for any $\varepsilon>0$, and thus, it represents a holomorphic function on the half-plane $\operatorname{Re}(s)>1$. By analytic continuation, $L(s, \chi)$ is extended to a meromorphic function on the whole complex $s$-plane. In particular, if $\chi=\chi^{0}$ (the principal character), then it reduces to the ordinary Riemann zeta function

$$
\zeta(s):=\sum_{n=1}^{\infty} \frac{1}{n^{s}}, \quad \operatorname{Re}(s)>1 .
$$

By analytic continuation, $\zeta(s)$ is also extended to a meromorphic function on the whole complex $s$-plane being holomorphic everywhere except for a simple pole at $s=1$ with residue 1 .

$M S C$ (2010): primary 11B37, 11B68, 11C20, 11M06; secondary 05A10, 11B75.

Keywords: Dirichlet $L$-function, Riemann zeta function, generalized Bernoulli numbers, recurrence relations, determinantal expressions. 
On the other hand, the Hurwitz zeta function $\zeta(s, c)$ is defined by the series

$$
\zeta(s, c):=\sum_{n=1}^{\infty} \frac{1}{(c+n)^{s}}, \quad \operatorname{Re}(s)>1, \operatorname{Re}(c) \notin\{-1,-2,-3, \ldots\} .
$$

This function converges absolutely for $\operatorname{Re}(s)=\sigma>1$ with uniform convergence in $\sigma \geq \sigma_{0}>1$; so that $\zeta(s, c)$ is an analytic function on the half-plane $\operatorname{Re}(s)>1$. As well as the Riemann zeta function, $\zeta(s, c)$ can be continued meromorphically to the whole complex $s$-plane with a simple pole at $s=1$ with residue 1 . In the case when $c=0$, this function coincides with $\zeta(s)$.

As is easily seen, $L(s, \chi)$ is represented by means of the Hurwitz zeta function. Indeed, we have

$$
\begin{aligned}
L(s, \chi) & =\sum_{a=1}^{f} \sum_{k=0}^{\infty} \frac{\chi(k f+a)}{(k f+a)^{s}}=\frac{1}{f^{s}} \sum_{a=1}^{f} \chi(a) \sum_{k=0}^{\infty}\left(k+\frac{a}{f}\right)^{-s} \\
& =\frac{1}{f^{s}} \sum_{a=1}^{f} \chi(a) \zeta\left(s, \frac{a}{f}\right),
\end{aligned}
$$

which is valid for all $s \in \mathbb{C}$.

Let $\Gamma$ be the gamma function defined by the improper integral

$$
\Gamma(z):=\int_{0}^{\infty} t^{z-1} e^{-t} d t=2 \int_{0}^{\infty} t^{2 z-1} e^{-t^{2}} d t, \quad \operatorname{Re}(z)>1,
$$

and $\tau(\chi)$ be the Gauss sum defined by

$$
\tau(\chi):=\sum_{a=1}^{f} \chi(a) e^{2 i \pi a / f} \quad(i:=\sqrt{-1}) .
$$

The gamma function $\Gamma$ can be extended by analytic continuation to a meromorphic function on $\mathbb{C}$ except for the simple poles $z=-n, n=0,1,2, \cdots$, with residues $(-1)^{n} / n$ !, and it follows that $\Gamma(z+1)=z \Gamma(z)$ for all $z \in \mathbb{C} \backslash\{0,-1,-2, \ldots\}$. In particular, we have $\Gamma(n)=(n-1)$ ! for an integer $n \geq 1$.

As is well-known, $L(s, \chi)$ satisfies the functional equation

$$
L(1-s, \chi)=\frac{f^{s-1} \Gamma(s)}{(2 \pi)^{s}}\left(e^{-i s \pi / 2}+\chi(-1) e^{i s \pi / 2}\right) \tau(\chi) L(s, \bar{\chi}),
$$

where $\bar{\chi}$ is the complex conjugate of $\chi$. There is an enormous amount of literature on this equation and related topics (for instance, see, e.g., $[6,8,9,19]$ ).

It is easy to show that $\Gamma(s)$ does not have zeros on the complex plane $\mathbb{C}$ and has only poles of order one at non-positive integers. Further, if $\chi$ is a primitive character, then $|\tau(\chi)|=\sqrt{f_{\chi}} \neq 0$. Since $L(s, \chi) \neq 0$ for $\operatorname{Re}(s)>0$, the above functional equation tells us that if $\operatorname{Re}(s)<0$, then $L(s, \chi)$ has only the so-called trivial zeros. That is to say, as well as $L(0, \chi)=0$ for a non-principal even character, we have for a primitive character $\chi$,

(i) $\chi(-1)=1 \Rightarrow L(-2 n, \chi)=0$ for all integers $n \geq 1$;

(ii) $\chi(-1)=-1 \Rightarrow L(1-2 n, \chi)=0$ for all integers $n \geq 1$.

Of course there are infinitely many zeros in the strip $\{s \in \mathbb{C} \mid 0<\operatorname{Re}(s)<1\}$, and also, the generalized Riemann hypothesis states that the Dirichlet $L$-functions 
have all their non-trivial zeros on the critical line $\operatorname{Re}(s)=1 / 2$. Incidentally, we would like to mention that it is widely believed that $L(1 / 2, \chi) \neq 0$ for 'all' primitive characters $\chi$, which was first conjectured by Chowla [13] in the case when $\chi$ is a quadratic character; but this problem remains still unsolved.

Let $B_{n, \chi}$ and $B_{n, \chi}(x), n=0,1,2, \ldots$, be the generalized Bernoulli numbers and polynomials attached to $\chi$ (a primitive Dirichlet character with conductor $f$ ). They are usually defined by means of the generating functions

$$
\begin{aligned}
\mathcal{F}_{\chi}(t) & :=\frac{1}{e^{f t}-1} \sum_{a=1}^{f} \chi(a) t e^{a t}=\sum_{n=0}^{\infty} \frac{B_{n, \chi} t^{n}}{n !}, \quad|t|<2 \pi / f ; \\
\mathcal{F}_{\chi}(t, x): & =\frac{1}{e^{f t}-1} \sum_{a=1}^{f} \chi(a) t e^{(x+a) t}=\sum_{n=0}^{\infty} \frac{B_{n, \chi}(x) t^{n}}{n !}, \quad|t|<2 \pi / f,
\end{aligned}
$$

respectively. From the functional identity $\mathcal{F}_{\chi}(t, x)=\mathcal{F}_{\chi}(t) e^{x t}$, it can be shown that $B_{n, \chi}(0)=B_{n, \chi}$ and $B_{n, \chi}(x)=\sum_{i=0}^{n}\left(\begin{array}{c}n \\ i\end{array}\right) B_{i, \chi} x^{n-i}$ for all integers $n \geq 0$.

Here it is clear that if $\chi \neq \chi^{0}$, then

$$
B_{0, \chi}=\frac{1}{f} \sum_{a=1}^{f} \chi(a)=0 .
$$

In addition, since $\mathcal{F}_{\chi}(t, x)=\chi(-1) \mathcal{F}_{\chi}(-t,-x)$, we immediately get

$$
B_{n, \chi}(x)=(-1)^{n} \chi(-1) B_{n, \chi}(-x),
$$

which shows us that if $n$ and $\chi$ have opposite parity; i.e., if $\chi(-1)=(-1)^{n+1}$, then $B_{n, \chi}=B_{n, \chi}(0)=0$ unless $n=f=1$. As is revealed from Proposition 1.1 below, this property is precisely consistent with (1.3).

Let $B_{n}$ and $B_{n}(x), n=0,1,2, \ldots$, be the classical Bernoulli numbers and polynomials defined by the generating functions

$$
\begin{gathered}
\mathcal{F}(t):=\frac{t}{e^{t}-1}=\sum_{n=0}^{\infty} \frac{B_{n} t^{n}}{n !}, \quad|t|<2 \pi ; \\
\mathcal{F}(t, x):=\frac{t e^{x t}}{e^{t}-1}=\sum_{n=0}^{\infty} \frac{B_{n}(x) t^{n}}{n !}, \quad|t|<2 \pi,
\end{gathered}
$$

respectively. Obviously, $B_{n} \in \mathbb{Q}$ and $B_{n}(x) \in \mathbb{Q}[x]$ for all integers $n \geq 0$. From the definitions of $B_{n, \chi}$ and $B_{n, \chi}(x)$ we see that if $\chi=\chi^{0}$, then

$$
\begin{aligned}
B_{n, \chi^{0}} & =\left\{\begin{array}{l}
-B_{1}=1 / 2 \text { for } n=1 ; \\
B_{n} \text { otherwise, }
\end{array}\right. \\
B_{n, \chi^{0}}(x) & =B_{n, \chi^{0}}(x+1)=\left\{\begin{array}{l}
1 \text { for } n=0 ; \\
B_{n}(x)+n x^{n-1} \text { otherwise. }
\end{array}\right.
\end{aligned}
$$

Further, since $f \mathcal{F}_{\chi}(t)=\sum_{a=1}^{f} \chi(a) \mathcal{F}(f t,(a-f) / f)$, one can express $B_{n, \chi}$ in terms of the values of classical Bernoulli polynomials, namely

$$
B_{n, \chi}=f^{n-1} \sum_{a=1}^{f} \chi(a) B_{n}\left(\frac{a-f}{f}\right) \quad(n \geq 0) .
$$


Next, we introduce a pivotal relation between generalized Bernoulli numbers and the values of $L(s, \chi)$ at non-positive integers. As a consequence of the fact that $-L(-n, \chi) / n$ ! for an integer $n \geq 0$ equals the residue of $\mathcal{F}_{\chi}(z) / z^{n+2}$ at $z=0$, we can state the following important formula:

Proposition 1.1. For an integer $n \geq 0$ it follows that

$$
L(-n, \chi)=-\frac{B_{n+1, \chi}}{n+1} .
$$

As is evident from (1.1) and (1.5), this formula is equivalent to

$$
L(-n, \chi)=f^{n} \sum_{a=1}^{f} \chi(a) \zeta\left(-n, \frac{a}{f}\right)=-\frac{f^{n}}{n+1} \sum_{a=1}^{f} \chi(a) B_{n+1}\left(\frac{a-f}{f}\right) .
$$

Similar to (1.6), the special values of the Riemann and Hurwitz zeta functions at non-positive integers are expressed by means of classical Bernoulli numbers and polynomials, respectively. Actually, it follows that for an integer $n \geq 0$,

$$
\text { (i) } \quad \zeta(-n)=(-1)^{n} \frac{B_{n+1}}{n+1} ; \quad \text { (ii) } \quad \zeta(-n, x)=-\frac{B_{n+1}(x)}{n+1} \text {. }
$$

In particular, we have $\zeta(0)=\zeta(0,1)=-1 / 2, \zeta(-2 n)=0$ for $n \geq 1$ (trivial zeros), and $\zeta(0, x)=-B_{1}(x)=1 / 2-x$. For the surrounding details on (1.6) and (1.7), see, e.g., $[7,18,26,29]$.

Among numerous number of recurrence relations for generalized Bernoulli numbers, the most basic linear one is

$$
\sum_{j=0}^{n-1}\left(\begin{array}{l}
n \\
j
\end{array}\right) \frac{B_{j+1, \chi}}{j+1} f^{n-j}+\frac{B_{0, \chi}}{n+1} f^{n+1}=\sum_{a=1}^{f} \chi(a) a^{n} \quad(n \geq 1),
$$

which is derived by expanding both sides of $\left(e^{f t}-1\right) \mathcal{F}_{\chi}(t)=t \sum_{a=1}^{f} \chi(a) e^{a t}$ into the Taylor power series and equating the coefficients of $t^{n+1}$ on both sides.

Using (1.6) and replacing $B_{0, \chi}$ by Kronecker's $\delta_{1 f}$ in view of $B_{0}=1$ and (1.4), one may convert (1.8) into the following recurrence relation for the values of $L(s, \chi)$ at non-positive integers:

$$
\sum_{j=0}^{n-1}\left(\begin{array}{l}
n \\
j
\end{array}\right) L(-j, \chi) f^{n-j}=-\sum_{a=1}^{f} \chi(a) a^{n}+\frac{f^{n+1}}{n+1} \delta_{1 f} \quad(n \geq 1) .
$$

This kind of formula has the disadvantage of demanding the previous knowledge of all the values of $L(s, \chi)$ at $s=0,-1, \ldots,-(n-1)$ to compute $L(-n, \chi)$.

In this paper we mainly study recurrence relations and determinantal expressions for the values of the Dirichlet $L$-function function at non-positive integers. In Section 2, applying a certain Saalschütz-Gelfand type formula for generalized Bernoulli numbers and based on (1.6), we derive some special recurrence formulas (that have a small number of terms) for the values of the Dirichlet $L$-function at non-positive integers. In Section 3 we discuss detreminantal expressions for these values using one of recurrences obtained in Section 1. In Section 4 we deal with several kinds of infinite sums constituted of the Riemann zeta values at negative odd integers and establish some closed-form expressions for these sums. In the 
final Section 5, as additional remarks, we give a very brief survey on the values of the Riemann zeta function at positive integers.

\section{RECURRENCE RELATIONS}

In this section we first introduce a certain Saalschütz-Gelfand type formula extended to generalized Bernoulli numbers and then convert it based on (1.6) into a recurrence relation for the values of the Dirichlet $L$-function at non-positive integers. Applying this recurrence, we then derive some concise formulas involving not only these values but also the Riemann zeta values at negative integers.

A large number of recurrence relations for classical Bernoulli numbers have been developed over the years (see, e.g., $[15,17,20,25]$ ). Among them, the best known linear one is

$$
B_{0}=1, \quad \sum_{j=0}^{n-1}\left(\begin{array}{l}
n \\
j
\end{array}\right) \frac{B_{j+1}}{j+1}+\frac{B_{0}}{n+1}=0 \quad(n \geq 1),
$$

which corresponds to (1.8) for $\chi=\chi^{0}$. At a first glance, we see that this identity involves all the consecutive Bernoulli numbers $B_{0}, B_{1}, \ldots, B_{n}$. In contrast to this, the following surprising and unusual recurrence relation was discovered first by Saalschütz [24] and later by M. B. Gelfand [16]:

$$
\sum_{i=0}^{k}\left(\begin{array}{l}
k \\
i
\end{array}\right) \frac{B_{m+1+i}}{m+1+i}+(-1)^{k+m} \sum_{j=0}^{m}\left(\begin{array}{c}
m \\
j
\end{array}\right) \frac{B_{k+1+j}}{k+1+j}=\frac{(-1)^{m+1}}{k+m+1}\left(\begin{array}{c}
k+m \\
k
\end{array}\right)^{-1},
$$

which is valid for all integers $k, m \geq 0$. The most distinguished feature of this identity is that the first $\min \{k, m\}$ Bernoulli numbers are completely missing unlike (2.1). These type formulas are usually called shortened (or incomplete) recurrence relations. The above Saalschütz-Gelfand identity can be extended in a natural way to Bernoulli polynomials and it is shown that

$$
\begin{gathered}
\sum_{i=0}^{k}\left(\begin{array}{l}
k \\
i
\end{array}\right) \frac{B_{m+1+i}(x)}{m+1+i}-\sum_{j=0}^{m}(-1)^{m+j}\left(\begin{array}{c}
m \\
j
\end{array}\right) \frac{B_{k+1+j}(x)}{k+1+j} \\
=x^{k}(x-1)^{m}+\frac{(-1)^{m+1}}{k+m+1}\left(\begin{array}{c}
k+m \\
k
\end{array}\right)^{-1} .
\end{gathered}
$$

A brief historical overview and full proofs of these formulas can be found, e.g., in $[2,5]$. Incidentally, we note that the polynomial sequence $\left\{B_{n}(x)\right\}_{n=0}^{\infty}$ forms an Appell sequence. That is, $B_{0}(x)=1 \neq 0$ and $\frac{d}{d x} B_{n+1}(x)=(n+1) B_{n}(x)$ for $n \geq 0$. Based on this fact, differentiate both sides of (2.2) twice with respect to $x$ and then set $x=0$ and $n=m-1=k-1$. Then we can deduce the classical von Ettingshausen-Stern identity discovered in the 18th century, namely

$$
\sum_{i=0}^{n+1}\left(\begin{array}{c}
n+1 \\
i
\end{array}\right)(n+i+1) B_{n+i}=0 \quad(n \geq 0)
$$

which involves only the second half of Bernoulli numbers up to $B_{2 n+1}$. 
Most recently, in [3] the present author extended (2.2) to generalized Bernoulli polynomials and proved the following shortened recurrence relation:

$$
\begin{gathered}
\sum_{i=0}^{k}\left(\begin{array}{c}
k \\
i
\end{array}\right) \frac{B_{m+1+i, \chi}(x)}{m+1+i}(q f)^{k-i}+\sum_{j=0}^{m}(-1)^{m+1+j}\left(\begin{array}{c}
m \\
j
\end{array}\right) \frac{B_{k+1+j, \chi}(x)}{k+1+j}(q f)^{m-j} \\
=\sum_{a=1}^{f} \chi(a) \sum_{r=0}^{q-1}(x+a+r f)^{k}(x+a+(r-q) f)^{m} \\
+\frac{(-1)^{m+1}}{k+m+1}\left(\begin{array}{c}
k+m \\
k
\end{array}\right)^{-1}(q f)^{k+m+1} \delta_{1 f},
\end{gathered}
$$

which is valid for arbitrary integers $k, m \geq 0$ and $q \geq 1$. If we set $x=0$ and $q=1$, then this identity is reduced to

$$
\begin{aligned}
& \sum_{i=0}^{k}\left(\begin{array}{c}
k \\
i
\end{array}\right) \frac{B_{m+1+i, \chi}}{m+1+i} f^{k-i}+\sum_{j=0}^{m}(-1)^{m+1+j}\left(\begin{array}{c}
m \\
j
\end{array}\right) \frac{B_{k+1+j, \chi}}{k+1+j} f^{m-j} \\
& \quad=\sum_{a=1}^{f} \chi(a) a^{k}(a-f)^{m}+\frac{(-1)^{m+1}}{k+m+1}\left(\begin{array}{c}
k+m \\
k
\end{array}\right)^{-1} f^{k+m+1} \delta_{1 f} .
\end{aligned}
$$

Furthermore, considering the special case for $m=k \geq 1,(2.3)$ leads to

$$
\begin{aligned}
& \sum_{r=0}^{k}\left(\begin{array}{l}
k \\
r
\end{array}\right)\left(1+(-1)^{k+1+r}\right) \frac{B_{k+1+r, \chi}}{k+1+r} f^{k-r} \\
& \quad=\sum_{a=1}^{f} \chi(a) a^{k}(a-f)^{k}+\frac{(-1)^{k+1}}{2 k+1}\left(\begin{array}{c}
2 k \\
k
\end{array}\right)^{-1} f^{2 k+1} \delta_{1 f} .
\end{aligned}
$$

Since the term involving $B_{k+1+r, \chi}$ on the left-hand side vanishes if $k+r$ is even, by observing only the case when $k+r$ is odd and then setting anew $k-r=2 j+1$ we obtain after dividing both sides by 2 ,

$$
\begin{aligned}
& \sum_{j=0}^{\lfloor(k-1) / 2\rfloor}\left(\begin{array}{c}
k \\
2 j+1
\end{array}\right) \frac{B_{2(k-j), \chi}}{2(k-j)} f^{2 j+1} \\
& \quad=\frac{1}{2} \sum_{a=1}^{f} \chi(a) a^{k}(a-f)^{k}+\frac{(-1)^{k+1}}{2(2 k+1)}\left(\begin{array}{c}
2 k \\
k
\end{array}\right)^{-1} f^{2 k+1} \delta_{1 f} .
\end{aligned}
$$

By converting (2.3) and (2.4) based on (1.6) into the recurrence relations for the values of the Dirichlet $L$-function at non-positive integers, we are able to establish the following theorem:

Theorem 2.1. For integers $k, m \geq 0$ we have

$$
\begin{gathered}
\sum_{i=0}^{k}\left(\begin{array}{c}
k \\
i
\end{array}\right) L(-m-i, \chi) f^{k-i}-\sum_{j=0}^{m}(-1)^{m+j}\left(\begin{array}{c}
m \\
j
\end{array}\right) L(-k-j, \chi) f^{m-j} \\
=-\sum_{a=1}^{f} \chi(a) a^{k}(a-f)^{m}+\frac{(-1)^{m}}{k+m+1}\left(\begin{array}{c}
k+m \\
k
\end{array}\right)^{-1} \delta_{1 f} .
\end{gathered}
$$


In particular, for an integer $k \geq 1$ we have

$$
\begin{aligned}
& \sum_{j=0}^{\lfloor(k-1) / 2\rfloor}\left(\begin{array}{c}
k \\
2 j+1
\end{array}\right) L(1-2(k-j), \chi) f^{2 j+1} \\
& \quad=-\frac{1}{2} \sum_{a=1}^{f} \chi(a) a^{k}(a-f)^{k}+\frac{(-1)^{k}}{2(2 k+1)}\left(\begin{array}{c}
2 k \\
k
\end{array}\right)^{-1} \delta_{1 f} .
\end{aligned}
$$

As is clearly seen, in order to compute $L(-n, \chi)(n \geq 1)$ recursively by using (2.5) or (2.6), we do not need the previous knowledge of all the values of $L(s, \chi)$ at $s=0,-1, \ldots,-(n-1)$ unlike (1.9). As a supplement, it should be noted that (2.6) has a substantial meaning only for an even character $\chi$. Otherwise, if $\chi$ is odd, then both sides of (2.6) completely vanish in view of (1.3) (ii), $\delta_{1 f}=0$, and

$$
\chi(a) a^{k}(a-f)^{k}+\chi(f-a)(f-a)^{k}(-a)^{k}=0, \quad a=1,2, \ldots,\lfloor f / 2\rfloor .
$$

The special case of Theorem 2.1 for $\chi=\chi^{0}$ leads immediately to the following corollary related to the Riemann zeta values at negative integers.

Corollary 2.2. For integers $k, m \geq 1$ we have

$$
\begin{aligned}
& \sum_{i=0}^{k}\left(\begin{array}{c}
k \\
i
\end{array}\right) \zeta(-m-i)+\sum_{j=0}^{m}(-1)^{m+j}\left(\begin{array}{c}
m \\
j
\end{array}\right) \zeta(-k-j) \\
& =\frac{(-1)^{m}}{k+m+1}\left(\begin{array}{c}
k+m \\
k
\end{array}\right)^{-1} .
\end{aligned}
$$

In particular, for $k \geq 1$ we have

$$
\sum_{j=0}^{\lfloor(k-1) / 2\rfloor}\left(\begin{array}{c}
k \\
2 j+1
\end{array}\right) \zeta(1-2 k+2 j)=\frac{(-1)^{k}}{2(2 k+1)}\left(\begin{array}{c}
2 k \\
k
\end{array}\right)^{-1}
$$

Note that nearly half of terms will disappear from the left-hand side of (2.7) because of $\zeta(-2 n)=0$ for all $n \geq 1$ as stated above.

\section{Determinantal EXPREssions}

In this section, by applying (2.6) we would like to deduce some determinantal expressions for $L(1-2 n, \chi)(n \geq 1)$ that seem to be quite new and original to the best of our knowledge. In what follows, we assume that $\chi$ is a primitive 'even' Dirichlet character with conductor $f$.

For simplicity's sake, let us denote $\mu_{k}:=\lfloor(k-1) / 2\rfloor(k \geq 1)$ and by $b_{k}$ the right-hand side of (2.6), namely

$$
b_{k}=b_{k, \chi}:=-\frac{1}{2} \sum_{a=1}^{f} \chi(a) a^{k}(a-f)^{k}+\frac{(-1)^{k}}{2(2 k+1)}\left(\begin{array}{c}
2 k \\
k
\end{array}\right)^{-1} \delta_{1 f} .
$$

Using these notations and the symmetric identity $\left(\begin{array}{c}m \\ i\end{array}\right)=\left(\begin{array}{c}m \\ m-i\end{array}\right)(0 \leq i \leq m)$, it is possible to rewrite (2.6) in the form

$$
\sum_{r=0}^{\mu_{k}}\left(\begin{array}{c}
k \\
k-1-2\left(\mu_{k}-r\right)
\end{array}\right) f^{1+2\left(\mu_{k}-r\right)} L\left(1-2 k+2\left(\mu_{k}-r\right), \chi\right)=b_{k},
$$


which is easily deduced by reversing the order of summation. As will be seen later, the form of (3.2) is much more convenient than that of (2.6) for our subsequent matrix discussion.

Following are concrete examples of (3.2) for the first few integers $k \geq 1$ :

$$
\begin{gathered}
\left(\begin{array}{l}
1 \\
0
\end{array}\right) f L(-1, \chi)=b_{1} ; \\
\left(\begin{array}{l}
2 \\
1
\end{array}\right) f L(-3, \chi)=b_{2} ; \\
\left(\begin{array}{c}
3 \\
0
\end{array}\right) f^{3} L(-3, \chi)+\left(\begin{array}{l}
3 \\
2
\end{array}\right) f L(-5, \chi)=b_{3} ; \\
\left(\begin{array}{l}
4 \\
1
\end{array}\right) f^{3} L(-5, \chi)+\left(\begin{array}{l}
4 \\
3
\end{array}\right) f L(-7, \chi)=b_{4} ; \\
\left(\begin{array}{l}
5 \\
0
\end{array}\right) f^{5} L(-5, \chi)+\left(\begin{array}{l}
5 \\
2
\end{array}\right) f^{3} L(-7, \chi)+\left(\begin{array}{l}
5 \\
4
\end{array}\right) f L(-9, \chi)=b_{5} ; \\
\left(\begin{array}{l}
6 \\
1
\end{array}\right) f^{5} L(-7, \chi)+\left(\begin{array}{l}
6 \\
3
\end{array}\right) f^{3} L(-9, \chi)+\left(\begin{array}{c}
6 \\
5
\end{array}\right) f L(-11, \chi)=b_{6} ; \\
\left(\begin{array}{l}
7 \\
0
\end{array}\right) f^{7} L(-7, \chi)+\left(\begin{array}{l}
7 \\
2
\end{array}\right) f^{5} L(-9, \chi)+\left(\begin{array}{l}
7 \\
4
\end{array}\right) f^{3} L(-11, \chi)+\left(\begin{array}{l}
7 \\
6
\end{array}\right) f L(-13, \chi)=b_{7} ; \\
\left(\begin{array}{l}
8 \\
1
\end{array}\right) f^{7} L(-9, \chi)+\left(\begin{array}{l}
8 \\
3
\end{array}\right) f^{5} L(-11, \chi)+\left(\begin{array}{l}
8 \\
5
\end{array}\right) f^{3} L(-13, \chi)+\left(\begin{array}{l}
8 \\
7
\end{array}\right) f L(-15, \chi)=b_{8} .
\end{gathered}
$$

For arbitrary fixed integer $n \geq 1$, taking $k=1,2, \ldots, n$ in (3.2), let us consider a system of $n$ linear equations in $n$ unknowns

$$
L(-1, \chi), L(-3, \chi), \ldots, L(1-2 n, \chi) .
$$

As is evident from (3.2), the coefficient matrix of this system is given by the lower triangular matrix

$$
A_{n, \chi}:=\left[\begin{array}{ccccc}
\boldsymbol{p}_{1} & & & & \\
& \boldsymbol{p}_{2} & & & O \\
& \boldsymbol{p}_{3} & & & \\
& & \boldsymbol{p}_{4} & & \\
& & \boldsymbol{p}_{5} & & \\
O & & & \ddots & \\
& & & & \boldsymbol{p}_{n}
\end{array}\right],
$$

where each $\boldsymbol{p}_{k}(1 \leq k \leq n)$ is a row vector of length $\lfloor(k+1) / 2\rfloor$ depending on $\chi$ (and thus, on $f$ ) such that

$$
\boldsymbol{p}_{k}=\boldsymbol{p}_{k, \chi}:=\left\{\begin{array}{l}
\left(\left(\begin{array}{l}
k \\
0
\end{array}\right) f^{k},\left(\begin{array}{l}
k \\
2
\end{array}\right) f^{k-2}, \ldots,\left(\begin{array}{c}
k \\
k-3
\end{array}\right) f^{3},\left(\begin{array}{c}
k \\
k-1
\end{array}\right) f\right) \text { if } k \text { is odd } \\
\left(\left(\begin{array}{l}
k \\
1
\end{array}\right) f^{k-1},\left(\begin{array}{l}
k \\
3
\end{array}\right) f^{k-3}, \ldots,\left(\begin{array}{c}
k \\
k-3
\end{array}\right) f^{3},\left(\begin{array}{c}
k \\
k-1
\end{array}\right) f\right) \text { if } k \text { is even. }
\end{array}\right.
$$

Denote by $\widetilde{A}_{n, \chi}$ the matrix replaced the last column of $A_{n, \chi}$ by $\left(b_{1}, b_{2}, \ldots, b_{n}\right)^{\mathrm{T}}$ (where ' $\mathrm{T}$ ' means the transpose of a vector), namely

$$
\widetilde{A}_{n, \chi}:=\left[\begin{array}{cccccc}
\boldsymbol{p}_{1} & & & & & b_{1} \\
& \boldsymbol{p}_{2} & & & b_{2} \\
& \boldsymbol{p}_{3} & & O & b_{3} \\
& & \boldsymbol{p}_{4} & & \cdot \\
& & \boldsymbol{p}_{5} & & \cdot \\
O & & & \ddots & & \cdot \\
& & & & \boldsymbol{p}_{n}^{*} & b_{n}
\end{array}\right],
$$


where $\boldsymbol{p}_{n}^{*}$ in the last row is the row vector of length $\mu_{n}$ obtained by removing the last entry $\left(\begin{array}{c}n \\ n-1\end{array}\right) f$ from $\boldsymbol{p}_{n}$. In particular, note that $\widetilde{A}_{1, \chi}=\left[b_{1}\right]$ and $\widetilde{A}_{2, \chi}=\left[\begin{array}{cc}1 & b_{1} \\ 0 & b_{2}\end{array}\right]$. Then, according to Cramer's rule, we obtain immediately

$$
L(1-2 n, \chi)=\frac{\operatorname{det} \widetilde{A}_{n, \chi}}{\operatorname{det} A_{n, \chi}} .
$$

Here we have $\operatorname{det} A_{n, \chi}=\prod_{k=1}^{n}\left(\begin{array}{c}k \\ k-1\end{array}\right) f=n ! f^{n}$, because the diagonal entries of $A_{n, \chi}$ are $\left(\begin{array}{l}1 \\ 0\end{array}\right) f,\left(\begin{array}{l}2 \\ 1\end{array}\right) f, \ldots,\left(\begin{array}{c}n \\ n-1\end{array}\right) f$. So that we can establish the following determinantal expression for $L(1-2 n, \chi)(n \geq 1)$ :

Theorem 3.1. For an integer $n \geq 1$ we have

$$
L(1-2 n, \chi)=\frac{\operatorname{det} \widetilde{A}_{n, \chi}}{n ! f^{n}} .
$$

Just to be sure, we repeat once again that this formula is meaningful only for an even character $\chi$. Otherwise, it turns out to be meaningless.

For further attempt, we now expand $\operatorname{det} \widetilde{A}_{n, \chi}$ along the first row. Since the first column of $\widetilde{A}_{n, \chi}$ is $\left(f, O_{n-1}\right)^{\mathrm{T}}$, where $O_{n-1}$ is the zero vector of length $n-1$, we see that the $(1, n)$ cofactor in $\operatorname{det} \widetilde{A}_{n, \chi}$ fully vanishes. Considering thereby the submatrix of $\widetilde{A}_{n, \chi}$ such that

$$
A_{n-1, \chi}^{*}:=\left[\begin{array}{ccccc}
\boldsymbol{p}_{2} & & & & b_{2} \\
\boldsymbol{p}_{3} & & & & b_{3} \\
& \boldsymbol{p}_{4} & & O & \cdot \\
& \boldsymbol{p}_{5} & & & \cdot \\
O & & \ddots & & \cdot \\
& & & \boldsymbol{p}_{n}^{*} & b_{n}
\end{array}\right] \quad(n \geq 2),
$$

we get $\operatorname{det} \widetilde{A}_{n, \chi}=f \cdot \operatorname{det} A_{n-1, \chi}^{*}$. Further, when $n \geq 2$, expanding $\operatorname{det} A_{n-1, \chi}^{*}$ along its last column $\left(b_{2}, b_{3}, \ldots, b_{n}\right)^{\mathrm{T}}$, we see that $n ! f^{n-1} L(1-2 n, \chi)$ can be represented by a linear combination of $b_{2}, b_{3}, \ldots, b_{n}$ over $\mathbb{Z}$, since all the cofactors of the last column entries are integers.

Consequently, letting $C_{k}$ be the $(k-1, n-1)$ cofactor in $\operatorname{det} A_{n-1, \chi}^{*}$ for each $k=2,3, \ldots, n$, we are led to the following determinantal expression for $L(1-2 n, \chi)$, which is a slightly advanced version of (3.3).

Theorem 3.2. Let $b_{k}$ be as in (3.1) and $n$ be an integer $\geq 2$. Then we have

$$
L(1-2 n, \chi)=\frac{\operatorname{det} A_{n-1, \chi}^{*}}{n ! f^{n-1}}=\frac{1}{n ! f^{n-1}} \sum_{k=2}^{n} C_{k} b_{k} .
$$

We now present some concrete examples of (3.4) for the first few integers $n \geq 2$. Cancelling common factors between the numerator and denominator, we have

$$
\begin{aligned}
& L(-3, \chi)=\frac{1}{2 f} b_{2} ; \\
& L(-5, \chi)=\frac{1}{6 f}\left(-f^{2} b_{2}+2 b_{3}\right)
\end{aligned}
$$




$$
\begin{aligned}
L(-7, \chi) & =\frac{1}{12 f}\left(2 f^{4} b_{2}-4 f^{2} b_{3}+3 b_{4}\right) \\
L(-9, \chi) & =\frac{1}{10 f}\left(-3 f^{6} b_{2}+6 f^{4} b_{3}-5 f^{2} b_{4}+2 b_{5}\right) \\
L(-11, \chi) & =\frac{1}{12 f}\left(10 f^{8} b_{2}-20 f^{6} b_{3}+17 f^{4} b_{4}-8 f^{2} b_{5}+2 b_{6}\right) .
\end{aligned}
$$

The special cases of (3.3) and (3.4) for $\chi=\chi^{0}$ yield the following determinantal expressions for the Riemann zeta values at negative integers, which are, however, just the translated ones of determinatal expressions for classical Bernoulli numbers that have been discussed in detail in [4].

Corollary 3.3. For brevity set $\widetilde{A}_{n}:=\widetilde{A}_{n, \chi^{0}}$ and $A_{n-1}^{*}:=A_{n-1, \chi^{0}}^{*}$. Then we have $\zeta(-1)=\operatorname{det} \widetilde{A}_{1}=b_{1, \chi^{0}}=-1 / 12$, and for an integer $n \geq 2$,

$$
\zeta(1-2 n)=\frac{\operatorname{det} \widetilde{A}_{n}}{n !}=\frac{\operatorname{det} A_{n-1}^{*}}{n !} .
$$

Needless to say, each $b_{k}=b_{k, \chi^{0}}$ appeared as an entry in the last columns of $\widetilde{A}_{n}$ and $A_{n-1}^{*}$ equals the right-hand side of (2.8), namely

$$
b_{k}=\frac{(-1)^{k}}{2(2 k+1)}\left(\begin{array}{c}
2 k \\
k
\end{array}\right)^{-1}
$$

Further, each row vector $\boldsymbol{p}_{k}=\boldsymbol{p}_{k, \chi^{0}}$ (resp. $\left.\boldsymbol{p}_{n}^{*}=\boldsymbol{p}_{n, \chi^{0}}^{*}\right)$ used in these matrices is, as a matter of course, given by setting $f=1$ in $\boldsymbol{p}_{k, \chi}\left(\right.$ resp. $\left.\boldsymbol{p}_{n, \chi}^{*}\right)$.

With the same notations as the above, we next define the square matrix $M_{n-2}$ of order $n-2$ by $M_{0}:=[0]$, and for $n \geq 3$,

$$
M_{n-2}:=\left[\begin{array}{ccccc}
3 & & & & b_{3} \\
\boldsymbol{p}_{4} & & O & & b_{4} \\
\boldsymbol{p}_{5} & & & & b_{5} \\
& \boldsymbol{p}_{6} & & & \cdot \\
& \boldsymbol{p}_{7} & & & \cdot \\
O & & \ddots & & \cdot \\
& & & \boldsymbol{p}_{n}^{*} & b_{n}
\end{array}\right] .
$$

Using this matrix, it was shown in [4, Corollary 3.5 (i)] that

$$
B_{2 n}+\frac{n(2 n-1)}{30} B_{2(n-1)}=-\frac{4 \operatorname{det} M_{n-2}}{(n-1) !} \quad(n \geq 2) .
$$

So that, using (1.7) (i) one can convey this to the following identity that shows an explicit relationship between the two adjacent Riemann zeta values at negative odd integers.

Corollary 3.4. For an integer $n \geq 2$ we have

$$
\zeta(1-2 n)+\frac{(n-1)(2 n-1)}{30} \zeta(3-2 n)=\frac{2 \operatorname{det} M_{n-2}}{n !} .
$$




\section{INFINITE SUMS}

In this section, making of (2.8), we derive some closed formulas for infinite sums involving the values of the Riemann zeta function at negative odd integers.

To begin with, we prepare the following infinite summation formulas related to the reciprocals of central binomial numbers.

Lemma 4.1. Let $\phi:=(1+\sqrt{5}) / 2$ (the golden ratio). Then it follows that

$$
\begin{aligned}
& \text { (i) } \sum_{k=0}^{\infty} \frac{1}{2 k+1}\left(\begin{array}{c}
2 k \\
k
\end{array}\right)^{-1}=\frac{2 \pi}{3 \sqrt{3}} ; \\
& \text { (ii) } \sum_{k=0}^{\infty} \frac{(-1)^{k}}{2 k+1}\left(\begin{array}{c}
2 k \\
k
\end{array}\right)^{-1}=\frac{4 \log \phi}{\sqrt{5}} ; \\
& \text { (iii) } \sum_{k=0}^{\infty} \frac{(-4)^{k}}{2 k+1}\left(\begin{array}{c}
2 k \\
k
\end{array}\right)^{-1}=-\frac{\log (\sqrt{2}-1)}{\sqrt{2}} .
\end{aligned}
$$

Proof. For any numbers $x, y \in \mathbb{C}$ with $\operatorname{Re}(x)>0$ and $\operatorname{Re}(y)>0$, let $B(x, y)$ be the (complete) beta function defined by

$$
B(x, y):=\int_{0}^{1} t^{x-1}(1-t)^{y-1} d t=\frac{\Gamma(x) \Gamma(y)}{\Gamma(x+y)}
$$

where $\Gamma$ is the gamma function defined and used in (1.2). Set here $x=y=k+1$ for an integer $k \geq 0$ to obtain

$$
B(k+1, k+1)=\frac{\Gamma(k+1) \Gamma(k+1)}{\Gamma(2 k+2)}=\frac{(k !)^{2}}{(2 k+1) !}=\frac{1}{2 k+1}\left(\begin{array}{c}
2 k \\
k
\end{array}\right)^{-1}
$$

Hence the infinite sum taken over $k=0,1,2, \ldots$ is given by

$$
\begin{aligned}
& \sum_{k=0}^{\infty} B(k+1, k+1)=\int_{0}^{1} \sum_{k=0}^{\infty} t^{k}(1-t)^{k} d t=\int_{0}^{1} \frac{d t}{1-t+t^{2}} \\
& =\int_{0}^{1} \frac{d t}{(t-1 / 2)^{2}+(\sqrt{3} / 2)^{2}}=\frac{2}{\sqrt{3}}\left[\tan ^{-1} \frac{2 t-1}{\sqrt{3}}\right]_{0}^{1}=\frac{2 \pi}{3 \sqrt{3}}
\end{aligned}
$$

which proves (i). In almost the same way as above, it can be shown that

$$
\begin{gathered}
\sum_{k=0}^{\infty}(-1)^{k} B(k+1, k+1)=\int_{0}^{1} \sum_{k=0}^{\infty}(-1)^{k} t^{k}(1-t)^{k} d t \\
=\int_{0}^{1} \frac{d t}{1+t-t^{2}}=\frac{4 \log \phi}{\sqrt{5}}
\end{gathered}
$$

and also

$$
\begin{gathered}
\sum_{k=0}^{\infty}(-4)^{k} B(k+1, k+1)=\int_{0}^{1} \sum_{k=0}^{\infty}(-4)^{k} t^{k}(1-t)^{k} d t \\
=\int_{0}^{1} \frac{d t}{1+4 t-4 t^{2}}=\frac{\log (\sqrt{2}-1)}{\sqrt{2}}
\end{gathered}
$$

which lead to (ii) and (iii), respectively. 
Note here that the formulas in (4.1) are not new. Indeed, we can find them in Sprugnoli's paper [27], in which he deduced a number of infinite summation formulas that involve the reciprocals of central binomial numbers by discovering the corresponding generating functions in terms of the arctangent function.

We now combine (2.8) and the formulas in (4.1) to obtain some closed-form expressions for infinite sums constituted of the Riemann zeta values at negative odd integers. Since $(2.8)$ is not valid for $k=0$, in order to make consistent with (2.8), divide both sides of each formula in (4.1) by 2 and then subtract the term value $1 / 2$ corresponding to $k=0$. By such manipulations we obtain

$$
\begin{aligned}
& \text { (i) } \sum_{k=1}^{\infty}(-1)^{k} \sum_{j=0}^{\lfloor(k-1) / 2\rfloor}\left(\begin{array}{c}
k \\
2 j+1
\end{array}\right) \zeta(1-2(k-j))=\frac{\pi}{3 \sqrt{3}}-\frac{1}{2} ; \\
& \text { (ii) } \sum_{k=1}^{\infty} \sum_{j=0}^{\lfloor(k-1) / 2\rfloor}\left(\begin{array}{c}
k \\
2 j+1
\end{array}\right) \zeta(1-2(k-j))=\frac{2 \log \phi}{\sqrt{5}}-\frac{1}{2} ; \\
& \text { (iii) } \sum_{k=1}^{\infty} 4^{k} \sum_{j=0}^{\lfloor(k-1) / 2\rfloor}\left(\begin{array}{c}
k \\
2 j+1
\end{array}\right) \zeta(1-2(k-j))=-\frac{\log (\sqrt{2}-1)}{2 \sqrt{2}}-\frac{1}{2} .
\end{aligned}
$$

Here we notice that each of these identities includes a plurality of the same zeta values on the left-hand side. So it is needed to gather them in one place to avoid such a duplication.

For this purpose, letting $N \geq 1$ be an arbitrary integer and $x$ be a real number, consider the finite sum

$$
S_{N}(x):=\sum_{k=1}^{N} x^{k} \sum_{j=0}^{\lfloor(k-1) / 2\rfloor}\left(\begin{array}{c}
k \\
2 j+1
\end{array}\right) \zeta(1-2(k-j)),
$$

and represent this as a linear combination of $\zeta(1-2 j), j=1,2, \ldots, N$; that is,

$$
S_{N}(x)=c_{1}(x) \zeta(-1)+c_{2}(x) \zeta(-3)+\cdots+c_{N}(x) \zeta(1-2 N),
$$

where every $c_{j}(x)$ is a polynomial function of $x$ with integer coefficients. We will now give an explicit expression of $c_{j}(x)$ by making use of the following weighted binomial sums. For integers $m, r$ with $m \geq r \geq 1$ let us define

$$
\alpha_{m, r}(x):=\sum_{i=0}^{r-1} x^{m+i}\left(\begin{array}{c}
m+i \\
2 i+1
\end{array}\right) .
$$

In particular, for the special case for $r=m$, write simply as $\alpha_{m}(x):=\alpha_{m, m}(x)$, i.e., in plain terms,

$$
\alpha_{m}(x):=x^{m}\left\{x^{0}\left(\begin{array}{c}
m \\
1
\end{array}\right)+x^{1}\left(\begin{array}{c}
m+1 \\
3
\end{array}\right)+\cdots+x^{m-1}\left(\begin{array}{c}
2 m-1 \\
2 m-1
\end{array}\right)\right\} .
$$

Using these weighted binomial sums, one is able to ascertain that

$$
c_{j}(x)=\left\{\begin{array}{l}
\alpha_{j}(x) \text { for } j=1,2, \ldots, \gamma_{N} \\
\alpha_{i, N+1-j}(x) \text { for } j=\gamma_{N}+1, \gamma_{N}+2, \ldots, N
\end{array}\right.
$$


where $\gamma_{N}:=\mu_{N}+1=\lfloor(N+1) / 2\rfloor$. Consequently, it is possible to write (4.3) precisely in the form

$$
S_{N}(x)=\sum_{j=1}^{\gamma_{N}} \alpha_{j}(x) \zeta(1-2 j)+\sum_{j=\gamma_{N}+1}^{N} \alpha_{j, N+1-j}(x) \zeta(1-2 j) .
$$

Here we may assert that as $N$ increases, the terms in the second sum on the right-hand side of (4.5) are embedded successively into the first sum. In fact, for any fixed integer $m \geq 1$ the coefficient $c_{m}(x)$ of $\zeta(1-2 m)$ grows step by step according to $0, \alpha_{m, 1}(x), \alpha_{m, 2}(x), \ldots, \alpha_{m, m-1}(x)$ and it finally arrives at

$$
\alpha_{m, m-1}(x)+x^{2 m-1}\left(\begin{array}{l}
2 m-1 \\
2 m-1
\end{array}\right)=\alpha_{m}(x) ;
$$

thus the term $\alpha_{m}(x) \zeta(1-2 m)$ would be incorporated into the first sum. For that reason, the limiting sum of $S_{N}(x)$ as $N \rightarrow \infty$ is given eventually by

$$
\lim _{N \rightarrow \infty} S_{N}(x)=\sum_{j=1}^{\infty} \alpha_{j}(x) \zeta(1-2 j)
$$

where the term involving $\zeta(1-2 j)$ for each $j \geq 1$ appears only once.

Based on this fact and taking $x=-1,1,4$ in (4.4), we are able to rewrite the identities in (4.2) in the following reasonable forms:

Theorem 4.2. For an integer $j \geq 1$ let $\alpha_{j}(x)$ be the weighted binomial sum defined in (4.4). Then we have

$$
\begin{aligned}
& \text { (i) } \sum_{j=1}^{\infty} \alpha_{j}(-1) \zeta(1-2 j)=\frac{2 \sqrt{3} \pi-9}{18} ; \\
& \text { (ii) } \sum_{j=1}^{\infty} \alpha_{j}(1) \zeta(1-2 j)=\frac{4 \sqrt{5} \log \phi-5}{10} ; \\
& \text { (iii) } \sum_{j=1}^{\infty} \alpha_{j}(4) \zeta(1-2 j)=-\frac{\sqrt{2} \log (\sqrt{2}-1)+2}{4} .
\end{aligned}
$$

\section{Additional Remarks}

In this final section, as additional remarks, we give a very brief survey on the values of the Riemann zeta function at 'positive' integers.

At the beginning, it may be worthwhile to mention that the famous Euler formula asserts for an integer $n \geq 1$,

$$
\zeta(2 n)=\sum_{k=1}^{\infty} \frac{1}{k^{2 n}}=(-1)^{n+1} \frac{(2 \pi)^{2 n}}{2(2 n) !} B_{2 n},
$$

which shows that $(-1)^{n+1} B_{2 n}>0$ and $\zeta(2 n) / \pi^{2 n}$ is a rational number. This formula can be obtained by comparing the coefficients of $z^{2 n}$ in the following two 
different kinds of infinite series expansions of $\pi z \cot (\pi z)$ in $|z|<1$ :

$$
\pi z \cot (\pi z)=\left\{\begin{array}{l}
1-2 \sum_{n=1}^{\infty} \zeta(2 n) z^{2 n} \\
1+\sum_{n=1}^{\infty} B_{2 n} \frac{(2 i \pi z)^{2 n}}{(2 n) !}(i:=\sqrt{-1}) .
\end{array}\right.
$$

As is well-known, the Riemann zeta function satisfies the functional equation

$$
\zeta(s)=\theta(s) \zeta(1-s)
$$

or equivalently,

$$
\zeta(1-s)=\theta(1-s) \zeta(s)
$$

for all $s \in \mathbb{C} \backslash\{0,1\}$, where $\theta(s)$ is the analytic function defined by

$$
\theta(s):=2(2 \pi)^{s-1} \sin (\pi s / 2) \Gamma(1-s) .
$$

Using this equation, one can evaluate $\zeta(1-2 n)$ by means of the value $\zeta(2 n)$, and vice versa. Indeed, it follows that

$$
\zeta(1-2 n)=\frac{2}{(2 \pi)^{2 n}} \sin (\pi(1-2 n) / 2) \Gamma(2 n) \zeta(2 n)=\frac{(-1)^{n} 2(2 n-1) !}{(2 \pi)^{2 n}} \zeta(2 n),
$$

which is consistent with (5.1) by way of (1.7) (i). We may therefore rephrase any formulas related to the Riemann zeta values at negative odd integers in terms of the values of $\zeta(s)$ at positive even integers if necessary. For example, considering the special case of (1.8) for $\chi=\chi^{0}$, replace $n$ by $2 n$ and then exclude the trivial zeros of $\zeta(s)$ from this identity. Then we get

$$
\sum_{k=0}^{n-1}\left(\begin{array}{c}
2 n \\
2 k+1
\end{array}\right) \zeta(1-2 n+2 k)=\frac{1-2 n}{2(2 n+1)} \quad(n \geq 1)
$$

Thus, by using (5.1) we obtain after some straightforward manipulation with the binomial coefficients and multiplying the whole by $(-1)^{n}(2 \pi)^{2 n} / 2(2 n)$ !,

$$
\sum_{k=0}^{n-1} \frac{(-1)^{k}(2 \pi)^{2 k}}{(2 k+1) !} \zeta(2 n-2 k)=\frac{(-1)^{n+1}(2 \pi)^{2 n}(2 n-1)}{4(2 n+1) !}
$$

In the same way as above, it is possible to rewrite the formulas (2.8), (3.5), (3.6), and (4.6) in terms of the Riemann zeta values at positive even integers.

What can we say about the positive odd case? In contrast to the even case, much less is known about the values $\zeta(2 n+1)$ for $n \geq 1$ despite all the efforts of many people that were being made. As the few known results, we can state that $\zeta(3)$ is irrational (Apéry's theorem in 1978) and there are infinitely many irrational numbers among the set $\{\zeta(2 n+1) \mid n=1,2,3, \ldots\}$ (Rivoal's theorem in 2000). In addition, Rivoal and Zudilin [23] recently showed that there exist at least two irrational numbers among the 33 odd zeta values $\zeta(5), \zeta(7), \ldots, \zeta(69)$. It is conjectured that the numbers $\zeta(2 n+1)$ and $\zeta(2 n+1) / \pi^{2 n+1}(n \geq 1)$ are both irrational; however, this still remains unsolved.

As is intimately associated with the above results, it has been conjectured for a long time that $\zeta(3), \zeta(5), \zeta(7), \ldots$ are algebraically independent over $\mathbb{Q}[\pi]$, but 
only very little is known about this problem. For immediate information, see, e.g., the legible textbook [22] by Ribenboim and the relevant references therein.

Concerning representations of $\zeta(2 n+1)$, there is no concise closed formula such as (5.1) up to the present, and it is usually represented by using infinite series or integrals. For example, it is well-known that (see, e.g., $[1,14]$ )

$$
\zeta(2 n+1)=\frac{(-1)^{n+1}(2 \pi)^{2 n+1}}{2(2 n+1) !} \int_{0}^{1} B_{2 n+1}(x) \cot (\pi x) d x
$$

which yields various infinite series representations through the Fourier expansion. Further, it should be specially mentioned that Lerch has established in 1901 the following fascinating formula. That is, for an odd integer $n \geq 1$,

$$
\zeta(2 n+1)=2^{2 n} \pi^{2 n+1} \sum_{k=0}^{n+1}(-1)^{k+1} \frac{B_{2 k}}{(2 k) !} \frac{B_{2 n+2-2 k}}{(2 n+2-2 k) !}-2 \sum_{k=1}^{\infty} \frac{k^{-(2 n+1)}}{e^{2 \pi k}-1},
$$

which involves an alternative convolution sum for Bernoulli numbers. This formula is a special case of the more general formula discovered by Ramanujan without proof and proved first by Malurkar in 1925 by using the Mellin transform. Note that the last summation on the right-hand side of (5.2) is actually expressed by means of a double infinite series involving Bernoulli numbers.

Most recently, Prévost [21, Corollary 4] proved the very plain and simple infinite series involving the Riemann zeta values at positive integers by applying the Padé approximation of the exponential function. Indeed, he derived

$$
\text { (i) } \sum_{k=0}^{\infty}(-1 / 4)^{k} \zeta(4 k+3)=1 ; \quad \text { (ii) } \sum_{k=0}^{\infty}(-1 / 4)^{k}(4 k+3) \zeta(4 k+4)=2 \text {. }
$$

Apart from these, Choi and Srivastava have proved various kinds of infinite series formulas that are constituted of the Riemann and Hurwitz zeta values at positive integers. For reference, see their book [28] and papers [10-12].

\section{REFERENCES}

[1] M. Abramowitz and I. Stegun (eds.), Handbook of Mathematical Functions with Formulas, Graphs and Mathematical Tables, Dover Publications, New York, 1972.

[2] T. Agoh, Recurrences for Bernoulli and Euler polynomials and numbers, Expositiones Mathematicae 18 (2000), 197-214.

[3] T. Agoh, Shortened recurrence relations for generalized Bernoulli polynomials, Journal of Number Theory 176 (2017), 149-173.

[4] T. Agoh, Determinantal expressions for Bernoulli polynomials, Integers: The Electronic Journal of Combinatorial Number Theory 19 (2019), \#A19, 23 pp.

[5] T. Agoh and K. Dilcher, Reciprocity relations for Bernoulli numbers, The American Mathematical Monthly 115 (2008), 237-244.

[6] T. M. Apostol, Dirichlet L-functions and character power sums, Journal of Number Theory 2 (1970), 223-234.

[7] T. M. Apostol, Introduction to Analytic Number Theory, Springer-Verlag, New York, 1976.

[8] B. C. Berndt, The functional equation of some Dirichlet series, Proceedings of the American Mathematical Society 29 (1971), 457-460.

[9] B. C. Berndt, The functional equation of some Dirichlet series, II, Proceedings of the American Mathematical Society 31 (1972), 24-26.

[10] J. Choi and H. M. Srivastava, Sums associated with the zeta function, Journal of Mathematical Analysis and Applications 206 (1997), 103-120. 
[11] J. Choi and H. M. Srivastava, A certain family of series associated with the zeta and related functions, Hiroshima Mathematical Journal 32 (2002), 417-429.

[12] J. Choi and H. M. Srivastava, Series involving the zeta functions and a family of generalized Goldbach-Euler series, The American Mathematical Monthly 121 (2014), 229-236.

[13] S. Chowla, The Riemann Hypothesis and Hilbert's Tenth Problem, Mathematics and Its Applications, Gordon and Breach Science Publishers, New York-London-Paris 1965.

[14] D. Cvijović and J. Klinowski, Integral representations of the Riemann zeta function for odd-integer arguments, Journal of Computational and Applied Mathematics 142 (2002), 435-439.

[15] K. Dilcher, Bernoulli and Euler polynomials, NIST Digital Library of Mathematical Functions, Chapter 24, Cambridge University Press, Cambridge-New York, 2010. Available online at: http://dlmf.nist.gov.

[16] M. B. Gelfand, A note on a certain relation among Bernoulli numbers (Russian), Bashkir. Gos. Univ. Uchen. Zap. Ser. Mat. 31 (1968), 215-216. 75-82.

[17] E. R. Hansen, A Table of Series and Products, Prentice-Hall, Inc., Englewood Cliffs, NJ, 1975.

[18] K. Iwasawa, Lectures on p-adic L-Functions, Annals of Mathematics Studies 74, Princeton University Press, 1972.

[19] A. Kuznetsov, On Dirichlet series and functional equations, Journal of Number Theory 180 (2017), 498-511.

[20] N. Nielsen, Traitéelementaire des nombres de Bernoulli, Gauthier-Villars, Paris, 1923.

[21] M. Prévost, Recurrence for values of the zeta function, Applied Numerical Mathematics 60 (2010), 1382-1394.

[22] P. Ribenboim, My Numbers, My Friends: Popular Lectures on Number Theory, SpringerVerlag, New York, 2000.

[23] T. Rivoal and W. Zudilin, A note on odd zeta values, Preprint, March 8, 2018. Available online at: arXiv:1803.03160v1 [math.NT].

[24] L. Saalschütz, Verkürzte Recursionsformeln für die Bernoullischen Zahlen, Zeitschrift für Mathematik und Physik 37 (1892), 374-378.

[25] L. Saalschütz, Vorlesungen über die Bernoullischen Zahlen, ihren Zusammenhang mit den Secanten-Coefficienten und ihre wichtigeren Anwendungen, Springer-Verlag, Berlin, 1893.

[26] G. Shimura, Elementary Dirichlet Series and Modular Forms, Springer Monographs in Mathematics, Springer-Verlag, New York, 2007.

[27] R. Sprugnoli, Sums of reciprocals of the central binomial coefficients, Integers: The Electronic Journal of Combinatorial Number Theory 6 (2006), \#A 27, 18 pp.

[28] H. M. Srivastava and J. Choi, Series Associated with the Zeta and Related Functions, Kluwer Academic Publishers, Dordrecht, Boston and London, 2001.

[29] L. C. Washington, Introduction to Cyclotomic Fields (2nd ed.), Graduate Texts in Mathematics 83, Springer-Verlag, New York, 1997.

Takashi Agoh, Department of Mathematics, Tokyo University of Science, Yamazaki, Noda, Chiba 278-8510, Japan

e-mail: agoh_takashi@ma.noda.tus.ac.jp 